\title{
Does Lower-Body BMD Develop at the Expense of Upper-Body BMD in Female Runners?
}

\author{
ALAN M. NEVILL ${ }^{1}$, MELONIE BURROWS ${ }^{2}$, ROGER L. HOLDER ${ }^{3}$, STEVE BIRD $^{4}$, and DAVID SIMPSON ${ }^{5}$ \\ ${ }^{1}$ University of Wolverhampton, School of Sport, Performing Arts, and Leisure, UNITED KINGDOM; ${ }^{2}$ University of \\ Exeter, School of Sport and Health Science, Exeter, UNITED KINGDOM; ${ }^{3}$ University of Birmingham, Edgbaston, \\ Birmingham, UNITED KINGDOM; ${ }^{4}$ Centre for Population Health, Sunshine Hospital and Melbourne University, \\ Victoria, AUSTRALIA; and ${ }^{5}$ Kent and Canterbury Hospital, Canterbury, UNITED KINGDOM
}

\begin{abstract}
NEVILL, A. M., M. BURROWS, R. L. HOLDER, S. BIRD, and D. SIMPSON. Does Lower-Body BMD Develop at the Expense of Upper-Body BMD in Female Runners? Med. Sci. Sports Exerc., Vol. 35, No. 10, pp. 1733-1739, 2003. Purpose: Evidence suggests that exercise plays an important role in stimulating site-specific bone mineral density (BMD). However, what is less well understood is how these benefits dissipate throughout the body. Hence, the purpose of the present study was to compare the levels of, and the correlation between, BMD recorded at 10 sites in female endurance runners, and to investigate possible determinants responsible for any inter-site differences observed. Methods: Repeated measures ANOVA was used to compare the BMD between sites and factor analysis was used to describe the pattern of intersite correlations. Allometric ANCOVA was used to identify the primary determinants of bone mass and how these varied between sites. Results: The ANOVA and factor analysis identified systematic differences in BMD between sites, with the greatest BMD being observed in the lower-body sites, in particular the legs. An investigation into the possible mechanisms responsible for these differences revealed "distances run" $\left(\mathrm{km} \cdot \mathrm{wk}^{-1}\right)$ as a positive, and "years of training" as a negative determinant of bone mass $(P<0.001)$. However, the effect of a number of determinants varied between sites $(P<0.05)$. Specifically, the ANCOVA identified that running further distances resulted in higher bone mass in the arms and legs. In contrast, training for additional years appeared to result in lower bone mass in the arms and lumber spine. Calcium intake was also found to be positively associated with bone mass in the legs but negatively associated at all other sites. Conclusions: A combination of running exercise and calcium intake would appear to stimulate the bone mass of women endurance runners at lower-body sites but at the expense of bone mass at upper-body sites. Key Words: BONE MINERAL DENSITY, INTERSITE CORRELATIONS, FACTOR ANALYSIS, PROPORTIONAL ALLOMETRIC REGRESSION MODEL, ANCOVA
\end{abstract}

$\mathrm{O}$ steoporosis is a bone disease associated with low bone mineral density (BMD) that increases the risk of debilitating bone fractures. Therefore, it is important to find effective intervention strategies for building bone and for preventing bone loss. The osteogenic stimulus provided by load-bearing exercise indicates it is an important lifestyle factor that could be used for the prevention of bone loss $(23,26)$. However, the specific role of physical activity in the maintenance or enhancement of bone mass or architecture remains elusive despite considerable research attention, with results inconsistent and inconclusive due to numerous methodological variations and limitations (30).

\footnotetext{
Address for correspondence: Professor Alan M. Nevill, University of Wolverhampton, School of Sport, Performing Arts and Leisure, Walsall Campus, Gorway Road, Walsall, WS1 3BD, United Kingdom; E-mail: a.m.nevill@wlv.ac.uk.

Submitted for publication February 2003.

Accepted for publication June 2003.
}

0195-9131/03/3510-1733

MEDICINE \& SCIENCE IN SPORTS \& EXERCISE E $_{\circledast}$

Copyright (C) 2003 by the American College of Sports Medicine

DOI: $10.1249 / 01 . M S S .0000089345 .28012 .23$
Physical activity has been shown to increase BMD in animals and human females by $0.9 \%$ per year in those limbs exercised (30). Indeed, strains on bone greater than needed for steady state remodeling will cause a modeling response that increases bone mass to meet the increasing load requirement (11). Various studies have concentrated on researching the effects of impact loading regimes across different sports on BMD and bone mineral content (BMC), and have suggested that the higher the impact load, the higher the BMD and BMC seen $(2,8,17,22,24)$. However, several studies have demonstrated that extremely high training loads can have a detrimental effect on bone (6) often via hormonal mechanisms $(10,12)$, whereas other studies have failed to demonstrate a relationship between training patterns and osteoporosis (18). These differences could be due to the fact that a minimum effective strain stimulus of mechanical loading is required to evoke an increase in the level of BMD, thus suggesting that high strain rates, distributed in unusual patterns, and short in duration and of sufficient frequency should be used to strengthen the bone (11).

It has also been suggested that this adaptive response to impact occurs only at the site of loading, indicating the site-specific nature of the bone remodeling response $(1,28)$. 
However, the majority of studies have only looked at BMD and BMC at a few bone sites, i.e., lumbar spine, hip, and radius (9), leaving incomplete the understanding of the effect of physical activity on bone health at skeletal sites throughout the body. In a recent study by Jorgensen et al. (14), it was concluded that there are significant differences in the classification of osteoporosis and osteopenia depending on the bone site measured. Moreover, McCarthy et al. (19) and Platen et al. (24) stated that the heterogeneity of response in the skeleton means that it is difficult to predict overall bone loss from measurements at one particular site. Indeed, Kanis (15) warns of discrepancies and misclassifications that can occur when diagnosing osteoporosis due to highly variable coefficients of variation (3) and inadequate level of correlation between BMD recorded at different skeletal sites throughout the body.

Given the site-specific nature of the bone remodeling response together with this evidence of inadequate correlations between skeletal sites, there would appear to be a lack of understanding of how the benefits of exercise at the loaded site dissipates to other sites throughout the body. Hence, the purpose of the present study was to compare the levels of, and the degree of correlation between, BMD recorded at 10 skeletal sites throughout the body in a group of female endurance runners. If significant and systematic differences in BMD are observed between sites, a proportional allometric regression model, originally proposed by Nevill et al. (20), will be used to identify the important determinants of bone mass and whether these determinants varied between skeletal sites, thus providing possible insight into the mechanisms for the observed differences in BMD between sites.

\section{MATERIALS AND METHODS}

Research design. The research was cross-sectional in design, allowing an investigation into the factors associated with, and differences between, bone mass recorded at 10 skeletal sites throughout the body in a population of female endurance runners of varying ages, diets, and training regimes.

Before initiating the study, the purposes and procedures were explained verbally and in writing to each participant, highlighting the possible risks and benefits associated with participation. Informed, written consent was obtained from all participants before any testing, in accordance with protocols defined by the local Kent and Canterbury Ethics Committee, which gave approval for the study.

Participants. Forty-nine female endurance runners (recreational to elite standard) were invited and agreed to participate in the study. To be accepted on the study the participants had to fulfill the following criteria: female, Caucasian, aged 18-44 yr (to decrease the possibility of women going through puberty and the menopause), and currently involved in endurance running. Participants were recruited from local and regional athletic clubs in the UK. Participants were asked to complete an osteoporosis risk factor and health questionnaire, before study commence-
TABLE 1. Personal characteristics and training details of the female endurance runners $(N=49)$.

\begin{tabular}{|c|c|}
\hline Variable & Female Endurance Runners \\
\hline Age (yr) & $30.8 \pm 5.6$ \\
\hline Stature $(\mathrm{cm})$ & $165 \pm 5.5$ \\
\hline Body mass $(\mathrm{kg})$ & $57.5 \pm 5.7$ \\
\hline Body fat $(\%)$ & $20.6 \pm 6.2$ \\
\hline Age at onset of training (yr) & $23 \pm 8$ \\
\hline Years training & $8.8 \pm 6$ \\
\hline Distance $\left(\mathrm{km} \cdot \mathrm{wk}^{-1}\right)$ & $32.3 \pm 17$ \\
\hline Training per week (h) & $8 \pm 5$ \\
\hline Sessions per week & $5 \pm 2$ \\
\hline Menarcheal age (yr) & $14 \pm 2$ \\
\hline Gynaecological age (yr) & $17 \pm 6$ \\
\hline Cycle frequency per year & $11 \pm 2$ \\
\hline Cycle flow (d) & $5 \pm 1$ \\
\hline OCA (months) & $56 \pm 54$ \\
\hline Eumenorrhoeic $(M)$ & 39 \\
\hline Oilgomenorrhoiec ( $M)$ & 10 \\
\hline Amenorrhoiec $(M)$ & 0 \\
\hline Average energy intake $\left(\mathrm{MJ} \cdot \mathrm{d}^{-1}\right)$ & $7.1 \pm 1.4$ \\
\hline Calcium $\left(\mathrm{mg} \cdot \mathrm{d}^{-1}\right)$ & $821 \pm 246$ \\
\hline Magnesium $\left(\mathrm{mg} \cdot \mathrm{d}^{-1}\right)$ & $254 \pm 58$ \\
\hline $\operatorname{Zinc}\left(\mathrm{mg} \cdot \mathrm{d}^{-1}\right)$ & $7.3 \pm 1.7$ \\
\hline Phosphorous (mg. $\mathrm{d}^{-1}$ ) & $1152 \pm 249$ \\
\hline
\end{tabular}

Values are mean $\pm S D$. Gynecological age is years since menarche; cycle flow is average length of menstrual flow; cycle frequency per year is average menstrual cycles experienced per year since menarche; OCA is length of oral contraceptive use in months.

ment, that evaluated their state of health. Participants were excluded from the study if they reported current or previous conditions that might interfere with bone metabolism, i.e., heart disease, long-term corticosteroid use, smoking, or alcoholism. Women currently using or who had previously used oral contraceptives were not excluded from the study.

The participants exercised on average $8 \mathrm{~h} \cdot \mathrm{wk}^{-1}(2-21 \mathrm{~h})$, at recreational to elite level for an average of $8 \mathrm{yr}(1-20 \mathrm{yr})$. The mean distance run per week was $32 \pm 17 \mathrm{~km}(8-112$ $\mathrm{km})$. In their training diaries, participants recorded comments on the type of training undertaken, which suggested that the training was predominantly steady runs, with a few upper- and lower-body weight-training sessions. However, the qualitative nature of such comments precluded any quantitative analysis.

Participants completed questionnaires to assess training, dietary, and menstrual status (amenorrheic $=0-3$ cycles a year; oligomenorrheic $=4-9$ cycles a year; eumenorrheic $\geq 10$ cycles a year). The questionnaires collected information on the history of menstruation, training and dietary status, as well as any recent changes (within the last year). A prospective, 7-d dietary record was also completed, from which average energy intake $\left(\mathrm{MJ} \cdot \mathrm{d}^{-1}\right)$, zinc $\left(\mathrm{mg} \cdot \mathrm{d}^{-1}\right)$, magnesium $\left(\mathrm{mg} \cdot \mathrm{d}^{-1}\right)$, phosphorous $\left(\mathrm{mg} \cdot \mathrm{d}^{-1}\right)$, and calcium $\left(\mathrm{mg} \cdot \mathrm{d}^{-1}\right)$ intake over this period was assessed (Dietmaster, version 4.0; Swift Computers Ltd., Phoenix, AZ). Refer to Table 1 for results.

BMD and body composition measurement. Participants were weighed (wearing minimal clothing) to the nearest $0.1 \mathrm{~kg}$ (Balance Beam Scale, Seca, Germany) and stature recorded to the nearest centimeter (stadiometer attached to Balance Beam Scale, Seca). BMC and density was measured at the lumbar spine (L2-L4), hip (femoral neck, Wards triangle, and greater trochanter), and across the whole body (legs, pelvis, thoracic spine, ribs, and arms), 
using dual energy x-ray absorptiometry (DEXA) (Hologic, QDR 4500w, Hologic Inc., Waltham, MA). Body fat percentage was also assessed during the whole-body scan. The standard Hologic protocol for positioning the lumbar spine, hip, and whole body was utilized. Bone mineral results were expressed as BMD $\left(\mathrm{g} \cdot \mathrm{cm}^{-2}\right)$. All scans were conducted and analyzed in the osteoporosis unit at the Kent and Canterbury Hospital. The in vivo precision of DEXA in the laboratory is $1 \%$ for the lumbar spine, hip, and whole body.

Statistical analysis. Differences in BMD between sites were compared using repeated-measures ANOVA. Bivariate correlations were used to assess the interrelationships between BMD recorded at 10 skeletal sites throughout the female runners' body. Factor analysis (using principal component analysis as the method of extraction) with varimax rotation was used to determine the minimum number and nature of the components or factors necessary to describe these correlations.

In an attempt to explain any differences in bone mass between sites and in the pattern of correlations, the following proportional allometric repeated-measures model adapted from Nevill et al. (20) was used to describe the important determinants of $\mathrm{BMC}\left(\mathrm{BMC}_{\mathrm{ij}}\right)$ at site $\mathrm{i}(\mathrm{i}=1,2$, $\ldots, 10)$ and participant $\mathrm{j}(\mathrm{j}=1,2, \ldots, 49)$, and how these might vary across the skeletal sites,

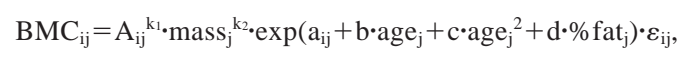

where for participant $\mathrm{j}, \mathrm{A}_{\mathrm{ij}}$ is the projected bone area of the skeletal site $\mathrm{i}$ being assessed, $\mathrm{a}_{\mathrm{ij}}$ is the constant intercept for each site, \%fat $t_{\mathrm{j}}$ is the whole-body fat (\%), and $\epsilon_{\mathrm{ij}}$ is the multiplicative error ratio. The model can be linearized with a log-transformation, and ANCOVA can then be used to estimate the differences due to "site" having controlled for differences in participants and other confounding covariates. The transformed log-linear ANCOVA model becomes,

$$
\begin{aligned}
& \log _{\mathrm{e}}\left(\mathrm{BMC}_{\mathrm{ij}}\right)= \\
& \mathrm{k}_{1} \cdot \log _{\mathrm{e}}\left(\mathrm{A}_{\mathrm{ij}}\right)+\mathrm{k}_{2} \cdot \log _{\mathrm{e}}\left(\operatorname{mass}_{\mathrm{j}}\right)+\mathrm{a}_{\mathrm{ij}}+\mathrm{b} \cdot \mathrm{age}_{\mathrm{j}}+\mathrm{c} \cdot \mathrm{age}_{\mathrm{j}}{ }^{2}+\mathrm{d} \cdot \% \mathrm{fat}_{\mathrm{j}}+\log _{\mathrm{e}}\left(\varepsilon_{\mathrm{ij}}\right) .
\end{aligned}
$$

Further determinants thought to be associated with BMC can be introduced into the model as additional covariates, e.g., average energy intake $\left(\mathrm{MJ} \cdot \mathrm{d}^{-1}\right)$, calcium $\left(\mathrm{mg} \cdot \mathrm{d}^{-1}\right)$ intake, distance run $\left(\mathrm{km} \cdot \mathrm{wk}^{-1}\right)$, and years of training, and ANCOVA can be used to assess whether any of the determinants varied from site to site, by introducing and testing the significance of a "covariate-by-site" interaction.

A significance level of 0.05 was accepted for all statistical procedures. If either the ANOVA or ANCOVA main effect difference between sites was detected, pairwise comparisons were made using the Bonferroni adjustment for multiple comparisons.

\section{RESULTS}

The personal characteristics and training details of the female runners are summarized in Table 1.

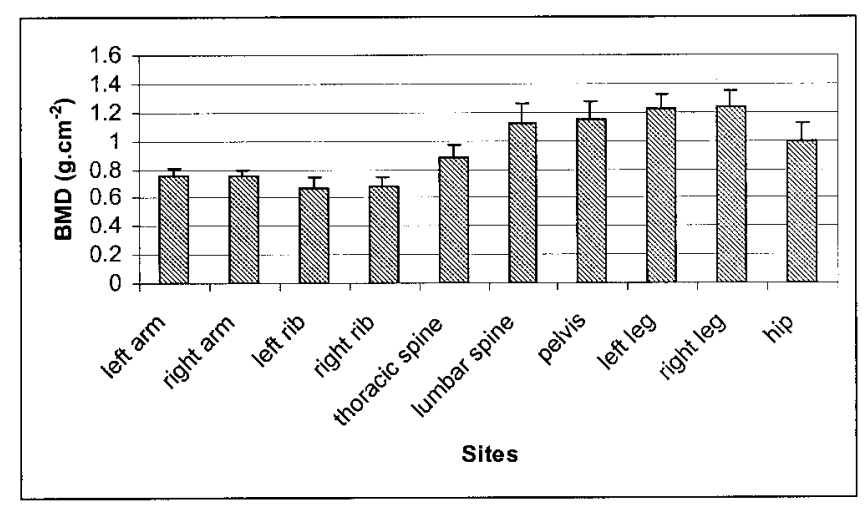

FIGURE 1-Differences in mean BMD $( \pm \mathrm{SD})$ by skeletal sites $(P<$ 0.001).

The repeated measures ANOVA of BMD identified a significant main effect due to sites $(P<0.001)$. The mean differences $( \pm \mathrm{SD})$ in BMD between sites can be seen in Figure 1. Bonferroni multiple comparison methods identified the legs as having the greatest BMD that was significantly greater than all other sites $(P<0.001)$. Due to the fact that bone at each site has a different shape, these comparisons in BMD should be interpreted with some caution, as explained in the Discussion.

All intersite correlations were significant $(P<0.05)$ with the exception of those between the left arm and left rib, thoracic spine and pelvis, denoted by "NS" (see Table 2).

The factor analysis suggested a two-factor solution, i.e., the 10 skeletal-site BMD variables were reduced to just two new underlying variables/factors or components. Using a convenient cut-off point of 0.55 , the factor loadings indicated a complete separation of sites into an upper- (ribs, right arm, thoracic spine, lumber spine, and pelvis) and lower-body (legs and hip) component. Together, these two components explained $71 \%$ of the variance with $59 \%$ being associated with the upper-body component. Interestingly, the left arm failed to identify strongly with either component. Note that if a less stringent cut-off point of 0.40 is used, the hip and thoracic spine sites load moderately on both components. Some of these patterns can be confirmed by examining the correlations in Table 2 .

The repeated measures ANCOVA of log-transformed BMC confirmed the significant main effect due to sites. Note that because there was no difference between adjusted BMC data for the right- and left-side of either the legs, arms or ribs, these data were collapsed into three "combined" BMC sites for the legs, arms, and ribs (see Fig. 1). The ANCOVA also identified significant determinants (covariates) as $\log _{\mathrm{e}}\left(\mathrm{A}_{\mathrm{i}}\right)$, age, age $\mathrm{e}^{2}$, distance run $\left(\mathrm{km} \cdot \mathrm{wk}^{-1}\right)$ and years of training. Note that the "site" and "subject" differences together with the covariates identified in the ANCOVA were able to explain $\mathrm{R}^{2}=99.4 \%$ of the variance in log-transformed BMC.

To assess whether the effect of the covariates varied across the different sites, each covariate was entered as a covariate-by-site interaction into the ANCOVA. The determinants age $(P=0.025)$, distance-run $(P=0.01)$, years- 
TABLE 2. Correlations between BMD recorded at 10 skeletal sites in female endurance runners $(N=49)$.

\begin{tabular}{|c|c|c|c|c|c|c|c|c|c|}
\hline BMD Site & $\begin{array}{l}\text { Right } \\
\text { Arm }\end{array}$ & $\begin{array}{l}\text { Left } \\
\text { Rib }\end{array}$ & $\begin{array}{l}\text { Right } \\
\text { Rib }\end{array}$ & $\begin{array}{c}\text { Thoracic } \\
\text { Spine }\end{array}$ & $\begin{array}{l}\text { Lumbar } \\
\text { Spine }\end{array}$ & Pelvis & $\begin{array}{l}\text { Left } \\
\text { Leg }\end{array}$ & $\begin{array}{l}\text { Right } \\
\text { Leg }\end{array}$ & Hip \\
\hline Left arm & 0.48 & $0.20^{\mathrm{NS}}$ & 0.29 & $0.21^{\mathrm{NS}}$ & 0.33 & $0.16^{\mathrm{NS}}$ & 0.30 & 0.38 & 0.31 \\
\hline Right arm & 1 & 0.53 & 0.66 & 0.55 & 0.70 & 0.62 & 0.46 & 0.49 & 0.63 \\
\hline Left rib & & 1 & 0.88 & 0.54 & 0.51 & 0.67 & 0.46 & 0.45 & 0.45 \\
\hline Right rib & & & 1 & 0.58 & 0.64 & 0.78 & 0.49 & 0.50 & 0.58 \\
\hline Thoracic spine & & & & 1 & 0.67 & 0.55 & 0.61 & 0.59 & 0.62 \\
\hline Lumbar spine & & & & & 1 & 0.72 & 0.44 & 0.48 & 0.69 \\
\hline Pelvis & & & & & & 1 & 0.39 & 0.40 & 0.55 \\
\hline Left leg & & & & & & & 1 & 0.89 & 0.75 \\
\hline Right leg & & & & & & & & 1 & 0.70 \\
\hline
\end{tabular}

All intersite correlations $(P<0.05)$ except those denoted by "NS" $(P>0.05)$.

of-training $(P<0.001)$, and calcium intake $(P=0.032)$ were found to have an effect on BMC, which varied significantly between sites. The fitted slope parameters of the covariates and their interactions are listed in Tables 3 and 4 , respectively. Note that the analysis estimated the slope parameters for all four interactions using the hip as the baseline site, and the deviations in the slopes of the other sites from the baseline hip are reported in the Table 4. With the addition of these interactions, the revised ANCOVA model was now able to explain $\mathrm{R}^{2}=99.5 \%$ of the variance in log-transformed BMC.

Interestingly, the strongest relationship between $\log _{\mathrm{e}}\left(\mathrm{BMC}_{\mathrm{i}}\right)$ and $\log$-transformed projected area, $\log _{\mathrm{e}}\left(\mathrm{A}_{\mathrm{i}}\right)$, appears to be linear (Fig. 2), confirmed by the estimated areal exponent, $\mathrm{k}_{1}$ being close to unity (see Table 3 ). This result is relevant to the method of normalizing BMC for differences in projected bone area, a finding that will be addressed later in the Discussion.

\section{DISCUSSION}

The ANOVA confirmed that the greatest BMD of the women endurance runners was in the legs, supporting the theory that the benefit of exercise is greatest at the loaded site. Figure 1 also confirmed that the upper-body sites of the arms, ribs, and, to a lesser extent, the thoracic spine, had the lowest mean BMD, suggesting that the level of BMD declines in sites the further they are located away from the loaded site. However, these findings are not entirely conclusive because comparing the BMD measurements recorded at different skeletal sites that have bones of different shapes, and in particular different bone depths or thicknesses, may result in misleading and incomparable bone densities. Nevertheless, the level of significance identified by the ANOVA of BMD provides strong evidence that the

TABLE 3. The estimated slope parameters/coefficients of the covariates from the log-transformed ANCOVA model (Eq. 2).

\begin{tabular}{lclcc}
\hline \multicolumn{1}{c}{ Term } & Coef & \multicolumn{1}{c}{ SEE } & \multicolumn{1}{c}{ T } & \multicolumn{1}{c}{$\boldsymbol{P}$} \\
\hline Constant & -0.789 & 0.267 & -2.95 & 0.001 \\
Inarea( $\mathrm{k}_{1}$ ) & 0.930 & 0.028 & 32.74 & $<0.001$ \\
Age (hip) & 0.083 & 0.018 & 4.51 & $<0.001$ \\
Age $^{2}$ & -0.0016 & 0.0003 & -4.81 & $<0.001$ \\
Distance (hip) $_{\text {Years-of-training (hip) }}$ & 0.0029 & 0.0007 & 4.0 & $<0.001$ \\
Calcium (hip) & -0.011 & 0.002 & -5.17 & $<0.001$ \\
\hline
\end{tabular}

SEE is the standard error of estimate, $\mathrm{T}$ is the "t-test" standard score, and $P$ is the associated probability based on Student's $t$ distribution. benefits of exercise is greatest at the loaded site and declines systematically in those sites the further they are located away from the loaded site.

Further evidence for this theory comes from the inter-site correlations. For the present population of women endurance runners, the factor analysis was unable to confirm a single factor (or principal component) necessary to describe the pattern of correlations. The factor analysis was able to identify two distinct components or factors that could be labeled as an upper-body and a lower-body component. The upper-body component consists of the ribs, right arm, thoracic spine, lumber spine and pelvis. The lower-body component contained both legs and the hip, with the left arm failing to identify strongly with either component. Although the hip did load moderately on the upper-body as well as strongly with the lower-body component, the factor analysis provides evidence that different mechanisms may be responsible for developing upper- and lower-body bone mass within this population of women endurance runners.

In order to understand what might cause the similarity within, and differences between, the upper- and lower-body skeletal sites, a proportional allometric ANCOVA model proposed by Nevill et al. (20) was used to identify any differences in $\mathrm{BMC}_{\mathrm{i}}$ between sites having controlled for the projected bone area $\left(\mathrm{A}_{\mathrm{i}}\right)$, body mass, age, whole-body fat $(\%)$, the distance run $\left(\mathrm{km} \cdot \mathrm{wk}^{-1}\right)$, and years of training. The ANOVA did indeed confirm that the legs had the greatest adjusted BMC that was greater than all other sites with the exception of the lumber spine and hip. However, as described above, these differences are not entirely conclusive because the bones have different shapes, and in particular, different depths, that may result in misleading and incomparable bone densities. On the other hand, identifying the important determinants of bone mass among these women endurance runners and how these determinants vary between sites is a valid method of identifying the likely mechanisms responsible for the observed mean and correlational differences in BMD between the upper- and lower-body skeletal sites.

As can be seen in Figure 2, the relationship between $\log _{\mathrm{e}}\left(\mathrm{BMC}_{\mathrm{i}}\right)$ and $\log$-transformed projected area, $\log _{\mathrm{e}}\left(\mathrm{A}_{\mathrm{i}}\right)$, is acceptably linear. This was confirmed by the fitted projected area exponent, $\mathrm{k}_{1}$ being approximately unity (see Table 3 ). This supports the use of the traditional BMD ratio $\mathrm{BMD}_{\mathrm{i}}=$ $\mathrm{BMC}_{\mathrm{i}} /\left(\mathrm{A}_{\mathrm{i}}\right)$ when attempting to normalize BMC for differences in projected bone area $\left(\mathrm{A}_{\mathrm{i}}\right)$. Interestingly, this areal 
TABLE 4. The estimated slope parameters/coefficients of the covariate-by-site interactions from the log-transformed ANCOVA model (Eq. 2).

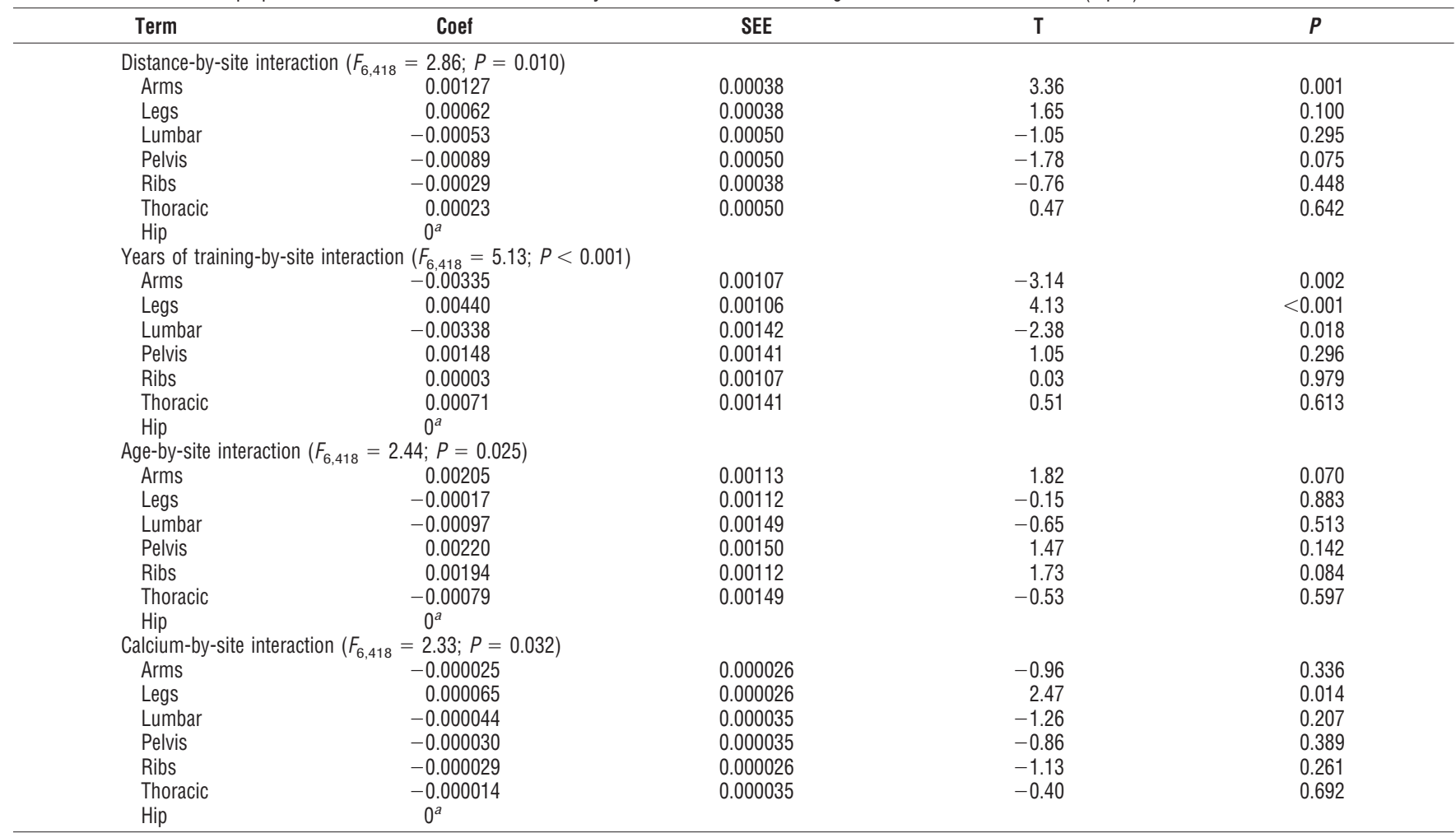

SEE is the standard error of estimate, $T$ is the "t-test" standard score, and $P$ is the associated probability based on Student's $t$ distribution.

${ }^{a}$ These parameters are set to zero because they are redundant.

BMD ratio has come under strong criticism by various authors $(7,16)$. These authors argue that if bone mass acquisition increases in proportion to an individual's skeletal bone volume, then theoretically the projected bone area, $\mathrm{A}_{\mathrm{p}}$, will not accurately reflect the skeletal bone volume being assessed. In an attempt to reduce the possible confounding effects of bone size, Carter and coworkers (7) recommend reporting an alternative ratio, referred to as the bone mineral apparent density (BMAD), estimated by the ratio $\mathrm{BMAD}=$ $\mathrm{BMC} /\left(\mathrm{A}_{\mathrm{p}}\right)^{3 / 2}\left(\mathrm{~g} \cdot \mathrm{cm}^{-3}\right)$, where $\left(\mathrm{A}_{\mathrm{p}}\right)^{3 / 2}$ is an estimate of the skeletal bone volume. Clearly, the results from this and a previous study using elite male athletes (21), provides compelling evidence that, certainly within such athletic popula-

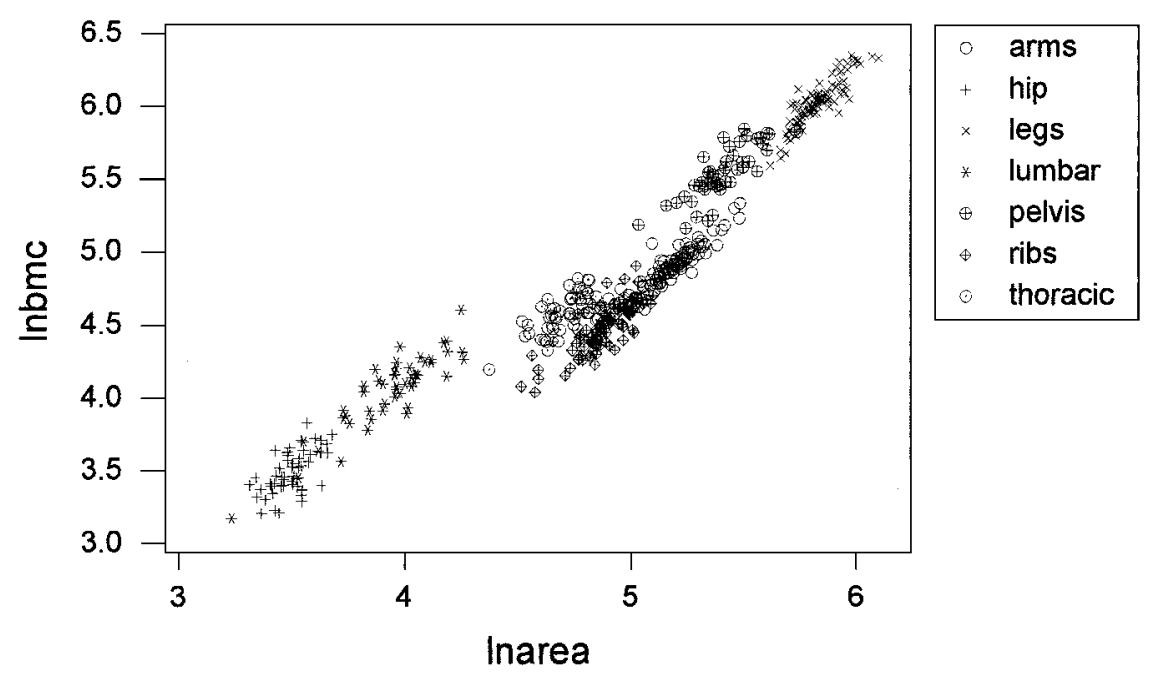

tions, BMC increases in proportion to the projected bone area $A_{p}$ rather than an estimate of the skeletal bone volume $\left(A_{p}\right)^{3 / 2}$, thus supporting the use of the traditional ratio BMD.

The variable age was entered into the ANCOVA model as a quadratic polynomial (using both the age and age ${ }^{2}$ terms), to accommodate the likelihood that bone mass peaks during mid-adolescence or early adulthood $(5,29)$. As anticipated, both covariate terms made a significant contribution to the model for bone mass. Using elementary differential calculus, the fitted age and age $^{2}$ parameters allow us to predict BMC (of the base-line site hip) will peak at the age of $(-b) /(2 c)=0.083 /(2 * 0.0016)=25.9$ yr. However, the significant age-by-site interaction confirms that bone mass 
at axial sites (e.g., lumber and thoracic spine) will peak earlier than more peripheral sites (e.g., arms and ribs) (4,27).

The overall positive association between adjusted BMC and distance run $\left(\mathrm{km}^{\circ} \mathrm{wk}^{-1}\right)$ might have been anticipated (the deviations from the positive baseline hip slope coefficient, reported in Table 4, all remain positive). Women runners that train by running greater distances are more likely to stimulate a positive osteogenic effect (25). The overall negative association between adjusted BMC and years of training (again the deviations from the negative baseline hip slope parameter, reported in Table 4, all remain negative) could simply reflect the lighter training loads of those dedicated women runners who have been running for many years, i.e., it is unlikely that women runners can sustain heavy training load indefinitely. Thus as the years of running progress, a dose-response relationship would be unlikely to result in sufficient force for bone remodelling (11).

When we investigated whether any of the above associations between BMC and those determinants identified in Table 3 varied across the different sites, the distance run $\left(\mathrm{km} \cdot \mathrm{wk}^{-1}\right)$, years of training, and calcium $\left(\mathrm{mg} \cdot \mathrm{d}^{-1}\right)$ intake were found to vary significantly between sites $(P<0.05)$. Having identified that running greater distances had an overall beneficial effect on BMC, the interaction term identified the BMC of the legs and arms to benefit from running longer distances compared with the other sites. The surprising benefit to the arms from running greater distances could be due to the extra muscle contractions that take place in the arms when running longer distances, compared with the shorter distances (23). In addition, those running greater distances tended to be the competitive athletes who combined mainly upper-body weight training in their regimes. Unfortunately, because details concerning upper-body weight training were only recorded in the women's diaries as qualitative comments, we were unable to confirm this effect using the ANCOVA analysis. We must acknowledge this as a limitation of the study and an area for future research.

Thus, in addition to the running (13), the weight training could have provided an effective osteogenic stimulus to the arms. Furthermore, having identified that bone mass declines with additional years of training at all skeletal sites, the adjusted BMC of the arms, and to a lesser extent the lumber spine, appears to decline at a greater rate compared

\section{REFERENCES}

1. American College of Sports Medicine. Medicine position stand: osteoporosis and exercise. Med. Sci. Sports Exerc. 27:I-vii, 1995.

2. Andreoli, A., M. Monteleone, M. Van Loan, L. Promenzio, U. TARANTINo, and A. DE LoREnZon. Effects of different sports on bone density and muscle mass in highly trained athletes. Med. Sci. Sports Exerc. 33:507-511, 2001.

3. Arlot, M. E., E. Sornay-Rendu, P. Garnero, B. Vey-Marty, and P. D. Delmas. Apparent pre- and postmenopausal bone loss evaluated by DXA at different skeletal sites in women: the OFELY cohort. J. Bone Miner. Res. 12:683-690, 1997.

4. Bass, S., P. D. Delmas, G. Pearce, E. Hendrich, A. Tabensky, and E. SeEman. The differing tempo of growth in bone size, mass and density in girls is region-specific. J. Clin. Invest. 104:795-804, 1999. with the other sites. Note that the rate of decline associated with additional years of training was least in the legs.

The other important finding of the present study was the differential effect of calcium intake on bone mass across the various skeletal sites, identified by the significant calciumby-site interaction $(P=0.032)$. Inspection of Tables 3 and 4 is able to confirm that there appears to be no benefit of calcium intake at the baseline site (hip). However, the calcium-by-site interaction revealed a positive association between calcium intake and BMC at the legs $(P<0.014)$ but a negative association at all other sites. This suggests that calcium intake is being diverted to the legs at the expense of all other skeletal sites. Note that none of the remaining dietary and menstrual-status covariates (average energy intake, zinc, magnesium, phosphorous, and menstrual cycles per year) nor the covariate-by-site interactions made a significant additional contribution to the ANCOVA analysis of log-transformed BMC.

In conclusion, these findings support previous research that suggests endurance running has a positive osteogenic effect on bone in lower-body skeletal sites and also supports the theory that bone mass acquisition obeys a principle of specificity (1). The present results also support the notion that although running longer distances are likely to stimulate bone mass, running for many years may be associated with lower levels in bone mass especially at upper-body sites. A possible mechanism for this result was identified when calcium intake was found to be positively associated with greater leg BMC but negatively associated with all other sites. Hence, although endurance running had a positive benefit to bone mass at the lower-body sites especially the legs, these benefits would appear to be at the expense of bone mass acquisition at some upper-body sites, where lower levels in bone mass were observed in dedicated women runners who trained for many years.

This research was made possible by the financial and technical support from the Kent and Canterbury Hospital, Osteoporosis Unit, Canterbury, Kent. The research team acknowledges the work of Dr. Michael O'Doherty (interpretation of BMD reports), Linda-Jane Archibold and Chris Murray (coordination and implementation of scans), and Chris Davies (coordination of bone densitometry scans). Finally, we should like to thank the reviewers and the Associate Editor for their helpful comments in revising the manuscript.

5. Bonjour, J. Q., G. Theintz, and B. Bucks. Critical years and stages of puberty for spinal and femoral bone mass accumulation during adolescence. J. Clin. Endocrinol. Metab. 73:555-563, 1991.

6. Burrows, M., A. M. Nevill S. Bird, and D. Simpson. Bone mineral status of female endurance runners. Br. J. Sports Med. 37:67-71, 2003.

7. Carter, D. R., M. L. Bouxsein, and R. Marcus. New approaches for interpreting projected bone densitometry data. J. Bone Miner. Res. 7:137-145; 1992.

8. Duncan, C. S., C. J. R. Blimkie, C. T. Cowell, S. T. Burke, J. N. Briody, R. Howman-Giles. Bone mineral density in adolescent female athletes: relationship to exercise type and muscle strength. Med. Sci. Sports Exerc. 34:286-294, 2002. 
9. Dyson, K, C. J. R. Blimkie, K. S. Davison, C. E. Webber, and J. D. ADACHI. Gymnastic training and bone density in pre-adolescent females. Med. Sci. Sports Exerc. 29:443-450, 1997.

10. Fehily, A. M., R. J. Coles, W. D. Evans, and P. C. Elwood. Factors affecting bone density in young adults. Am. J. Clin. Nutr. 56:579-586, 1992.

11. Frost, H. M. Muscle, bone and the Utah paradigm: a 1999 review. Med. Sci. Sports Exerc. 32:911-917, 2000.

12. Grimston, S. K., N. D. Willows, and D. A. Hanley. Mechanical loading regime and its relationship to bone mineral density in children. Med. Sci. Sports Exerc. 25:1203-1210, 1993.

13. Heinonen, A., P. OJa, P. Kannus, H. Haapasalo, A. Manttari, and I. VUORI. Bone mineral density in female athletes representing sports with different loading characteristics of the skeleton. Bone 17:197-203, 1995.

14. Jorgensen, H. L., L. Warming, N. H. Buarnason, P. B. Andersen, and C. Hassager. How does quantitative ultrasound compare to dual X-ray absorptiometry at various skeletal sites in relation to the WHO diagnosis categories? Clin. Physiol. 21:51-59, 2001.

15. Kanis, J. A. Diagnosis of osteoporosis and assessment of fracture risk. Lancet 359:1929-1936, 2002.

16. Katzman, D. K., L. K. Bachrach, D. R. Carter, and R. Marcus. Clinical and anthropometric correlates of bone mineral acquisition in healthy adolescent girls. J. Clin. Endocrinol. Metab. 73:13321339, 1991.

17. Lima, F., D. Falco, J. Baima, J. G. Carazzato, and R. M. R. Pereira. Effect of impact load and active load on bone metabolism and body composition of adolescent athletes. Med. Sci. Sports Exerc. 33:1318-1323, 2001

18. Marcus, R., C. CAnN, and P. Madvig. Menstrual function and bone mass in elite women distance runners: endocrine and metabolic factors. Ann. Int. Med. 102:158-163, 1985.

19. Mccarthy, I., A. Goodship, R. Herzog, V. Oganov, E. Stussi, and M. VAHLENSIECK. Investigation of bone changes in microgravity during long and short duration space flight: comparison of techniques. Eur. J. Clin. Invest. 30:1044-1054, 2000.
20. Nevill, A. M., R. L. Holder, N. Maffuldi, et al. Adjusting bone mass for differences in projected bone area and other confounding variables: an allometric perspective. J. Bone Miner. Res. 17:703708, 2002

21. Nevill, A. M., R. L. Holder, and A. D. Stewart. Modeling elite male athletes' peripheral bone mass, assessed using regional dual x-ray absorptiometry. Bone 32:62-68, 2003.

22. Nickolos-Richardson, S. M., C. M. Modlesky, P. J. O'Connor, and R. D. LEWIs. Premenarcheal gymnasts posses higher bone mineral density than controls. Med. Sci. Sports Exerc. 32:63-69, 2000.

23. Pettersson, U., H. Alfredson, P. Nordstrom, K. HenrikssonLARSEN, and R. LORENTZON. Bone mass in female cross country skiers: relationship between muscle strength and different bone mineral density sites. Calcif. Tissue Int. 67:199-206, 2000.

24. Platen, P., E. H. Chae, R. Antz, R. Lehmann, J. Kuehlmorgen, and B. AlloLio. Bone mineral density in top level male athletes of different sports. Eur. J. Sport Sci. 1:108-120, 2001.

25. Rubin, C. T., and L. E. LANYON. Regulation of bone mass by mechanical strain magnitude. Calcif. Tissue Int. 37:411-417, 1985.

26. Sandstrom, P., P. Jonsson, R. Lorentzon, and K. Thorsen. Bone mineral density and muscle strength in female ice hockey players. J. Sports Med. 21:524-528, 2000.

27. Seeman, E. Pathogenesis of bone fragility in women and men. Lancet 359:1841-1850, 2002.

28. Stewart, A. D., and J. Hannan. Total and regional bone density in male runners, cyclists, and controls. Med. Sci. Sports Exerc. 32:1373-1377, 2000.

29. Vico, L., B. Prallet, D. Chappard, B. Pallot-Prades, R. Pupier, and C. Alexandre. Contributions of chronological age, age at menarche and menopause and of anthropometric parameters to axial and peripheral bone densities. Osteoporos. Int. 2:153-158, 1992.

30. Wolff, I., J. J. Croonenborg, C. G. Kemper, P. J. Kostense, and J. W. R. Twisk. The effect of exercise training programs on bone mass: a meta-analysis of published controlled trials in pre- and postmenopausal women. Osteoporos. Int. 9:1-12, 1999. 